\title{
Uma Ferramenta de Apoio ao Gerenciamento de Padrões para Propósitos Pedagógicos
}

\author{
Josias Marques da Silva ${ }^{1}$, Ellen Francine Barbosa ${ }^{2}$, Maria Lydia Fioravanti², \\ Aracele Garcia de Oliveira Fassbinder ${ }^{1,2}$ \\ ${ }^{1}$ Instituto Federal de Educação, Ciência e Tecnologia do Sul de Minas Gerais \\ (IFSULDEMINAS), Campus Muzambinho, Muzambinho - MG - Brasil \\ ${ }^{2}$ Instituto de Ciências Matemáticas e de Computação (ICMC), \\ Universidade de São Paulo (USP), São Carlos - SP - Brasil \\ josiasmarquesareado@gmail.com, francine@icmc.usp.br, \\ mlfioravanti@usp.br, aracele.garciadifsuldeminas.edu.br
}

Resumo. Neste trabalho, um protótipo web de apoio à criação, compartilhamento e reuso de práticas de ensino descritas na forma de padrões educacionais é apresentado. Um padrão, de forma geral, contém soluções recorrentes para problemas que ocorrem frequentemente, dentro de um determinado contexto. Ele segue uma estrutura pré-definida que facilita o diálogo e a comunicação entre as pessoas envolvidas. Dessa forma, professores, tutores, projetistas instrucionais, dentre outros, podem transformar conhecimento tácito oriundo de experiências e práticas educacionais pessoais em padrões que também podem ser compartilhados e utilizados por todos.

\section{Cenário de uso}

Mais do que domínio e competência sobre um determinado assunto, professores precisam ser capazes de buscar, conhecer e, sempre que possível, criar novas práticas de ensino. Entretanto, buscar e conhecer práticas de ensino criadas por outros educadores requer que as mesmas estejam documentadas e disponíveis em algum meio (site, livro, etc.). Já a criação, muitas vezes, está relacionada com o conhecimento tácito, ou seja, adquirido a partir de experiências bem-sucedidas que um professor, nesse caso, acumulou no decorrer da sua carreira. Para que o ciclo continue, é importante que esse conhecimento tácito seja formalizado e disponibilizado de alguma forma, promovendo, assim, o reuso de práticas que também serão úteis para outros professores. Diante disso, surge a necessidade de encontrar formas de facilitar o armazenamento e compartilhamento de práticas de ensino entre professores novatos e experientes.

De forma geral, um padrão descreve uma solução para um problema que ocorre com frequência dentro de um determinado contexto, podendo ser considerado um par "problema/solução", de acordo com Buschmann et al. (1996). O conceito foi inicialmente proposto pelo arquiteto Christopher Alexander (Alexander et al., 1977), para o domínio de arquitetura e construção de cidades. Desde então, padrões têm sido muito utilizados 
em vários âmbitos do conhecimento. $\mathrm{Na}$ área de computação, eles têm sido utilizados no apoio ao desenvolvimento de software. Já no contexto educacional, existem os Padrões Pedagógicos, do inglês Pedagogical Patterns. Para Eckstein et. al. (2001), padrões pedagógicos são formas de documentar experiências de docência, podendo ser compartilhados com outros docentes, oferecendo soluções já testadas para serem reutilizadas.

Os padrões podem existir isoladamente ou de forma agrupada, por exemplo formando linguagens de padrões relacionados a fim de cumprir um determinado objetivo. Uma linguagem de padrão implica que o seu conjunto de padrões cobre os aspectos mais importantes em um domínio particular. Uma linguagem de padrão para a aplicação da estratégia ativa denominada Aprendizagem Baseada em Problemas, por exemplo, deve estar completa, ou seja, devem existir padrões que tratam boa parte dos aspectos da construção e implementação dessa estratégia.

Visando facilitar o gerenciamento e compartilhamento de padrões, ferramentas também têm sido desenvolvidas. UI-Patterns ${ }^{1}$, por exemplo, é um repositório de padrões de interface de software e permite aos usuários visualizarem padrões voltados para esse fim. Já o Pedagogical Pattern Collector ${ }^{2}$, projeto desenvolvido por pesquisadores ingleses, permite que educadores explorem, criem ou adaptem conjuntos de padrões pedagógicos (planos de aulas, estratégias de aprendizagem, experimentos, dentre outros).

Embora padrões sejam úteis para armazenar e registrar conhecimento tácito, padrões pedagógicos e ferramentas que apoiem a prática docente, principalmente na língua portuguesa, ainda são pouco explorados pela comunidade científica de computação aplicada à educação.

Considerando as questões pontuadas anteriormente, este trabalho descreve o protótipo de uma ferramenta web que possibilita a criação, o gerenciamento e o compartilhamento de padrões pedagógicos, tanto para reuso quanto para modificações/melhorias. A ferramenta pode ser utilizada por educadores, tutores, projetistas instrucionais, dentre outros, independentemente do contexto, área ou disciplina de atuação.

Este trabalho está organizado da seguinte forma. A Seção 2 apresenta o processo de desenvolvimento adotado para construir o protótipo de apoio ao gerenciamento de padrões educacionais, bem como as tecnologias de softwares utilizadas. A Seção 3 apresenta as principais funcionalidades do protótipo. Considerações, lições aprendidas e trabalhos futuros são descritos na Seção 4.

\footnotetext{
${ }^{1}$ http://www.ld-grid.org/resources/tools/pedagogical-pattern-collector

2 http://ui-patterns.com/
} 
VI Congresso Brasileiro de Informática na Educação (CBIE 2017)

Anais dos Workshops do VI Congresso Brasileiro de Informática na Educação (WCBIE 2017)

\section{Desenvolvimento do Protótipo}

Para o desenvolvimento do protótipo, foram utilizadas práticas e métodos ágeis, tais como o conceito de Minimum Viable Product (MVP) e Kanban, bem como a linguagem PHP, utilizando o framework frontend Bootstrap na versão 3.3.7, o framework backend Codeigniter na versão 3.1.4 e o banco de dados MySQL na versão 6.3.

Os conceitos apresentados no livro "Direto ao ponto: Criando produtos de forma enxuta", de Caroli (2016), foram aplicados para definir a visão do protótipo e o seu Produto Mínimo Viável (MVP), conforme detalhado a seguir.

Inicialmente, foi definida a visão do produto:

Para Educadores, tutores, projetistas instrucionais, dentre outros.

Interessados em ferramentas para gerenciar e/ou buscar padrões de práticas pedagógicas; ou que precisam registrar conhecimento tácito sobre o design de práticas pedagógicas e também compartilhá-las;

o IF-Patterns

é um software web

que permite catalogar e compartilhar padrões de experiências ou práticas de docência, independente da área de atuação ou nível acadêmico,

Diferentemente das ferramentas de repositórios de padrões de software, que permite o catálogo relacionado ao desenvolvimento de software, disponíveis geralmente em inglês; e diferente de estratégias de divulgação de planos de aula.

o nosso produto será desenvolvido em língua portuguesa e permite o catálogo de padrões pedagógicos.

Em seguida, a equipe discutiu e definiu o que o produto é ou faz, em contraste com o que ele não é ou não faz:

\section{O produto é:}

- Um repositório de padrões pedagógicos voltados para registrar conhecimento tácito, melhores soluções e experiências oriundas de práticas de ensino;

- Uma ferramenta web multiplataforma.

\section{O produto não é:}

- Um repositório para outros tipos padrões (ex.: software, arquitetura);

- Um repositório de conteúdo de disciplinas;

- Um repositório no formato de planos de aulas.

\section{O produto faz (permite):}

- login;

- criação de novos padrões;

- classificação dos padrões visíveis ao público ou não;

- permite relacionamentos entre padrões;

- permite agrupamento dos padrões;

- customização dos padrões por terceiros;

- avaliação dos padrões.

O produto não faz (não permite):

- disponibilização de conteúdos de disciplinas; 
VI Congresso Brasileiro de Informática na Educação (CBIE 2017)

Anais dos Workshops do VI Congresso Brasileiro de Informática na Educação (WCBIE 2017)

Também foram definidos os objetivos, as personas, as funcionalidades inicialmente previstas, o entendimento técnico e de negócio, os riscos, os fluxos de interação (passos para uma persona atingir um objetivo) e, por fim, o planejamento das versões mínimas (MVPs) a serem entregues de forma contínua, conforme destacado na Tabela 1:

\begin{tabular}{|l|c|}
\hline \multicolumn{1}{|c|}{ Funcionalidades } & MVPs \\
\hline Tela principal de apresentação. & 1 \\
\hline Login. & 1 \\
\hline Tela inicial para visualização das linguagens. & 1 \\
\hline Gerenciar Linguagens (inserir, excluir, alterar, listar). & 1 \\
\hline Gerenciar categorias. & 1 \\
\hline Gerenciar Padrões (inserir, excluir, alterar, listar). & 1 \\
\hline Cadastro de docentes. & 2 \\
\hline $\begin{array}{l}\text { Opção disponível para definir relacionamentos no ato da criação e na customização (pré-definidos: } \\
\text { complementa, antecessor, sucessor). }\end{array}$ & 2 \\
\hline Opção de avaliação e comentários nos padrões. & 2 \\
\hline Opção de busca por padrão, linguagem, autor, meus padrões. & 2 \\
\hline
\end{tabular}

Tabela 1: Identificando as Versões Mínimas do Protótipo (MVPs).

O protótipo tem sido aplicado em um contexto real, para fins de validação. Foi inicialmente testado com padrões pedagógicos de design de aprendizagem para Cursos Online Abertos e Massivos (MOOCs) e, também, padrões para especificação de requisitos pedagógicos de aplicações móveis educacionais. Também está disponível para uso por outros educadores que queiram compartilhar práticas e experiências pedagógicas.

\section{Apresentação do Protótipo}

O protótipo está disponível para acesso no endereço:

- labsoft.muz.ifsuldeminas.edu.br/projetos/openedupatterns

- login: teste@teste

- senha: teste

Já o vídeo demonstrativo pode ser acessado no youtube:

- https://www.youtube.com/watch?v=7v6AxIsf6Bg\&rel=0 
VI Congresso Brasileiro de Informática na Educação (CBIE 2017)

Anais dos Workshops do VI Congresso Brasileiro de Informática na Educação (WCBIE 2017)

Conforme definição da MVP 1 (Tabela 1), as principais funcionalidades do protótipo descrito neste trabalho são apresentadas a seguir.

A página inicial apresenta algumas informações e as linguagens públicas já criadas por outros usuários (Figura 1):

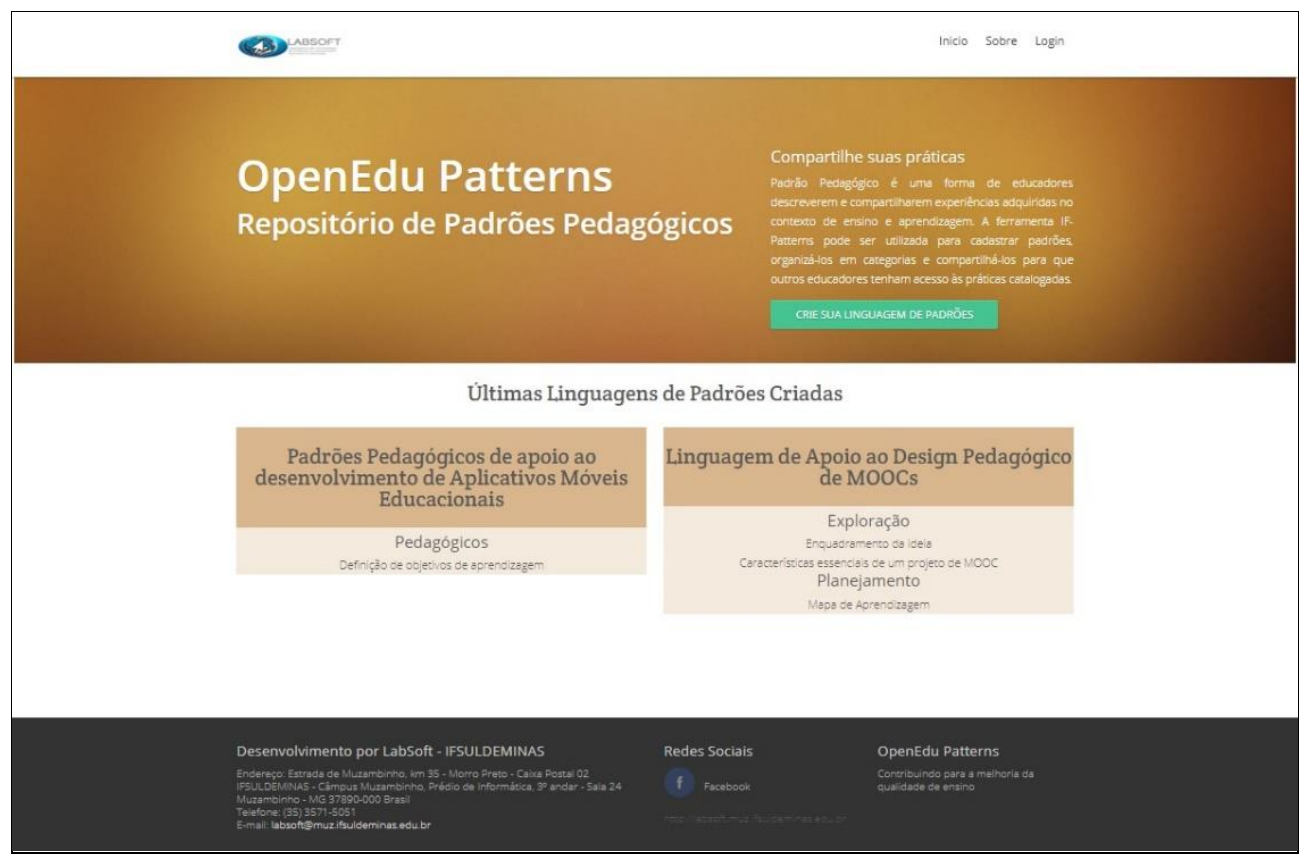

Figura 1 - Página Inicial do protótipo.

Ao se efetuar o login, por exemplo usando os dados teste@teste e a senha teste, são exibidas as linguagens já inseridas pelo usuário autenticado na ferramenta (Figura 2).

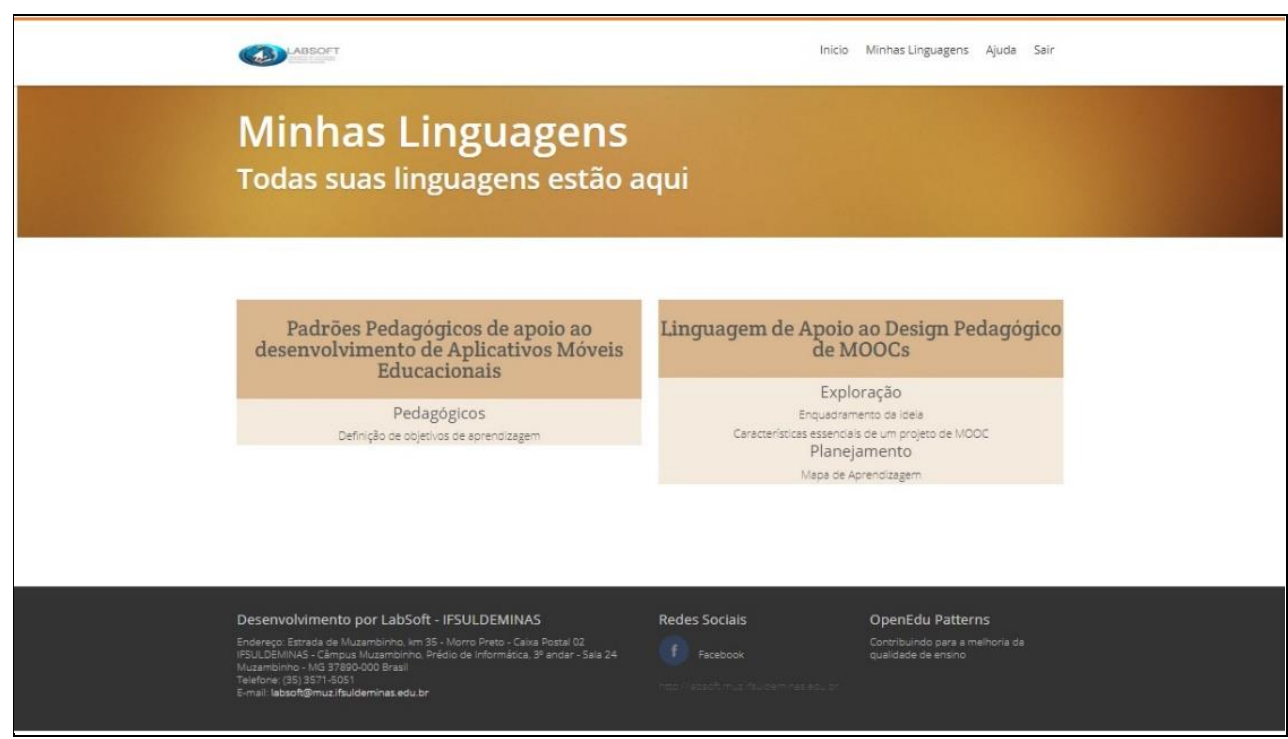

Figura 2 - Tela de "Minhas linguagens". 
VI Congresso Brasileiro de Informática na Educação (CBIE 2017)

Anais dos Workshops do VI Congresso Brasileiro de Informática na Educação (WCBIE 2017)

Inicialmente, é necessário criar uma linguagem de padrões, fornecendo o nome, o tipo da visibilidade (pública ou privada) e uma breve descrição da mesma (Figura 3). A visibilidade privada pode ser usada no caso de padrões ainda em construção e validação pelos autores. O ideal é deixá-los públicos para acesso geral.

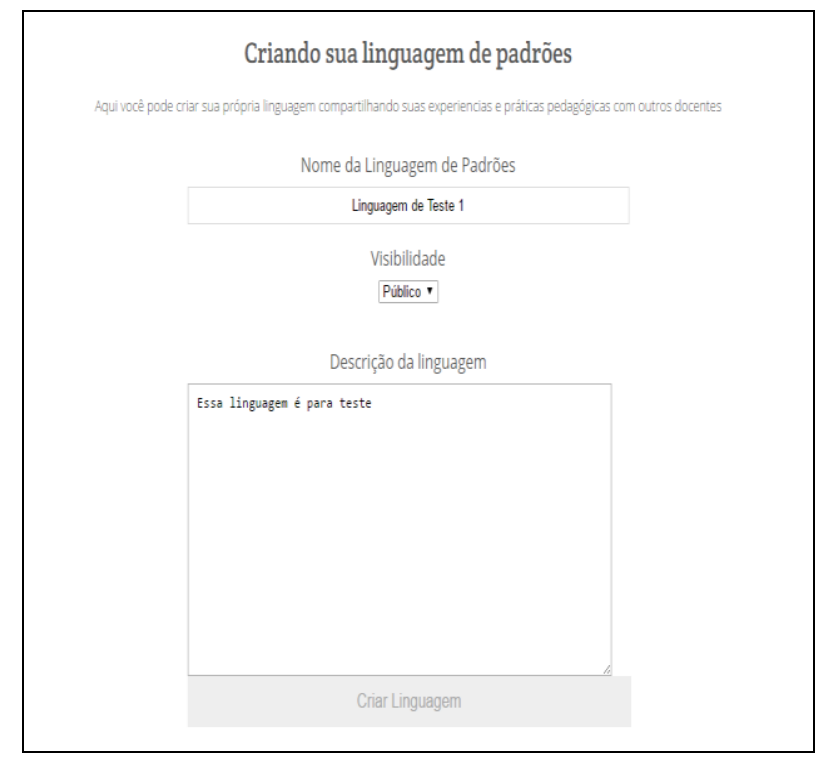

Figura 3 - Tela de criação de linguagem de padrões.

Em seguida, pode-se acrescentar padrões à linguagem criada. Na versão atual da ferramenta, um conjunto pré-definido de campos devem ser preenchidos: nome, categoria, contexto, problema e solução (Figura 4).

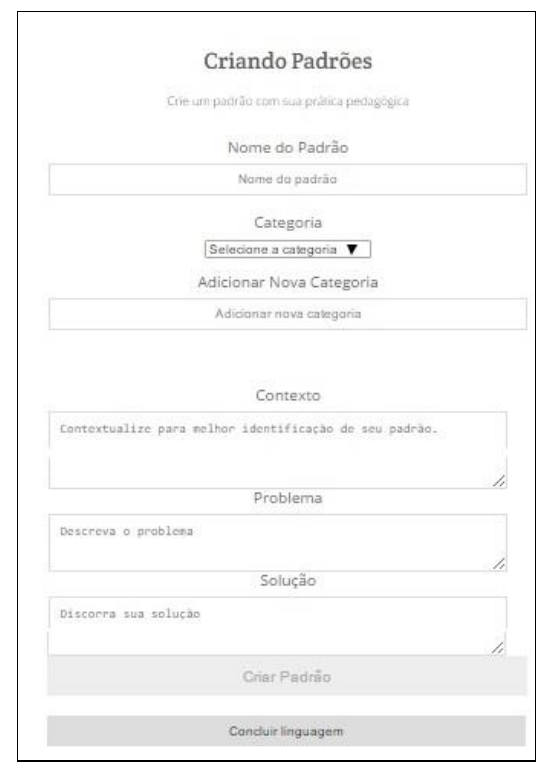

Figura 4 - Tela de criação de padrões. 
VI Congresso Brasileiro de Informática na Educação (CBIE 2017)

Anais dos Workshops do VI Congresso Brasileiro de Informática na Educação (WCBIE 2017)

Também é possível visualizar os dados individuais de cada padrão já cadastrado (Figura 5).

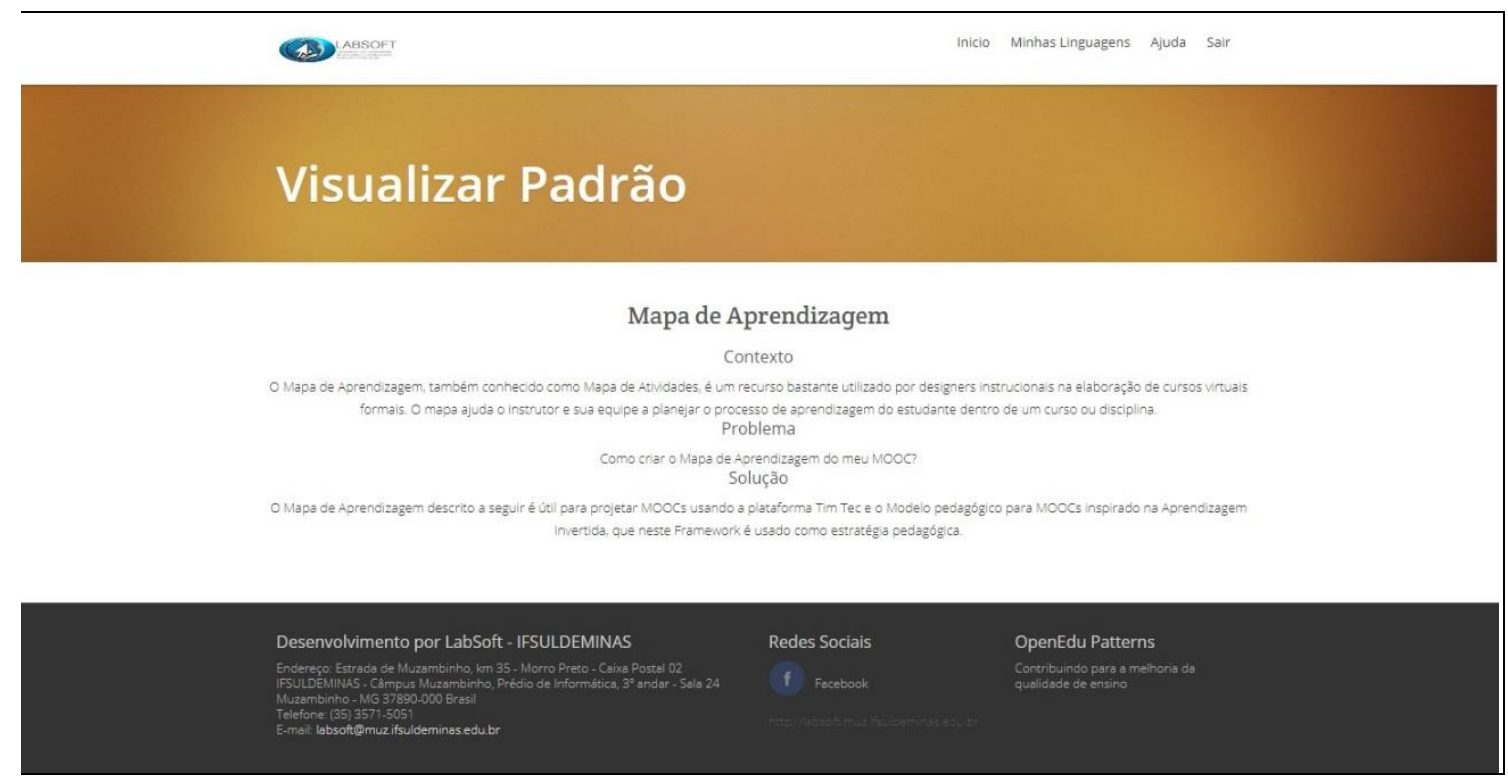

Figura 5 - Tela de exibição de um padrão.

\section{Considerações finais}

Este trabalho descreve o protótipo de uma ferramenta web que está sendo desenvolvida para uso por educadores e demais interessados no gerenciamento e compartilhamento de padrões para propósitos pedagógicos.

Espera-se, com isso, estimular a criação de práticas de ensino inovadoras e a sua posterior formalização por meio da escrita de padrões e seus relacionamentos e agrupamentos para que linguagens de padrões sejam constituídas.

Considerando o uso de práticas ágeis baseadas no desenvolvimento iterativo e incremental, a construção e validação da ferramenta envolve entregas contínuas baseadas em versões mínimas do produto definidas por meio da ideia de MVP. Dessa forma, atividades futuras envolvem a implementação das demais funcionalidades e validação da ferramenta em si.

\section{Agradecimentos}

Os autores agradecem o IFSULDEMINAS Campus Muzambinho e os professores Paulo César dos Santos e Diego Saqui pelas contribuições dadas durante o desenvolvimento do protótipo descrito neste trabalho, bem como ao IFSULDEMINAS Campus Muzambinho. 
VI Congresso Brasileiro de Informática na Educação (CBIE 2017)

Anais dos Workshops do VI Congresso Brasileiro de Informática na Educação (WCBIE 2017)

\section{References}

Alexander, C., Ishikawa, S., Silverstein, M., Jacobson, M., Fiksdahl-King, I., Angel, S. (1977) “A pattern language: Towns, buildings, construction", New York: Oxford University Press. Disponível em: http://library.uniteddiversity.coop/Ecological_Building/A_Pattern_Language.pdf. Acesso em 19 jun. 2017.

Buschmann, F., Meunier, R., Rohnert, H., Sommerlad, P., Stal, M. (1996) "PatternOriented Software Architecture: A System of Patterns", Chichester, England: John Wiley \& Sons.

Caroli, P. (2016) "Direto ao ponto: Criando Produtos de Forma Enxuta”, São Paulo: Casa do Código.

Eckstein, J., Manns, M. L., Voelter, M. (2001) "Pedagogical patterns: capturing best practice in teaching object technology", Software Focus, 2(1), pp. 9-12. 